\title{
Rethinking the Bacterial Genetic Regulation
}

\section{Sylvie Reverchon ${ }^{1}$, Patrick Sobetzko ${ }^{2 *}$, William Nasser ${ }^{1}$ and Georgi Muskhelishvili, ${ }^{1,2}$}

${ }^{1}$ Microbiologie, Adaptation, Pathogénie, UMR5240 CNRS-UCBL-INSA-Bayer Crop Science, Lyon, France

2Jacobs University Bremen, D-28759 Bremen, Germany

Bacteria are the most ancient and abundant organisms on the earth. Whereas bacterial organisms served as first cellular model systems for explorations of genetic control by approaches of molecular biology, our understanding of bacterial gene regulatory mechanisms is still far from complete. Yet, deep insights into genetic regulation are urgently required due to the increased frequency of nosocomial infections caused by multidrug-resistant bacteria [1], as well as increased agricultural damage caused by the bacterial plant pathogens $[2,3]$.

In most general terms, understanding of bacterial genetic regulation needs the knowledge of mechanisms coordinating the interactions between the regulatory factors (proteins or small RNA molecules) and the structural entities (genes or groups of genes) harboring the genetic functions. The prerequisite for this is the knowledge of all the regulatory factors and the mechanisms coordinating their inputs. This complexity of organization presents a profound methodological problem.

However, recent studies implementing high-throughput approaches to study the genetic regulation system may provide a breakthrough leading to a paradigm shift in the field. First of all, advances of the experimental technology and associated bioinformatics tools revealed widespread antisense transcription in the genome [4], as well as new levels of spatial organization of genes beyond the classical operon structure [5,6], which dominated the field for the last five decades. The bacterial genome is assumed to be organized in topologically isolated domains of about $10 \mathrm{~kb}$ size on average [7]. The functional role of these topological domains remains unclear, yet it is revealing, that the relative spatial organization of the transcription units in the genome appears to play an important role in mediating genetic regulation by relaying the DNA supercoil dynamics induced by translocating transcription machineries to neighbor genes over distances $(\geq 10 \mathrm{~kb})$, substantially exceeding the size of individual operons $[8,9]$. Furthermore, extended genomic spatial transcript patterns have been observed that cannot be readily explained on the basis of classical transcription factor (TF) -target gene (TG) interactions [5,9]. Recent studies made it increasingly evident that regulatory mechanisms based on spatial proximity and orientation of genes are evolutionarily conserved [6] and at least as important, as those mediated by TF-TG interactions $[10,11]$.

Obviously, genetic control based on spatial proximity of genes depends on the configuration of the DNA, which in turn depends on the supercoiling level and structural dynamics of the chromosome. Recent studies suggest a high degree of structural organisation of the bacterial chromosome. Various spatial organisation patterns revealed in E. coli include the polarity of chromosomal Ori and Ter ends [12], rrn functional domain spanning the chromosomal Ori end [13], megabasesized macrodomains [14], 200-900 kb size transient structuralfunctional domains [9], periodic patterns of regulated genes [15], clusters of nucleoid-perturbation sensitive genes [16], spatial transcript patterns spanning regions of 16 to $800 \mathrm{~kb}$ size [5], $33 \mathrm{~kb}$ size functional domains of "core genes" [17], 30-50 kb size "folding domains" [18], 10$20 \mathrm{~kb}$ topological domains [7] and 5-10 kb size gene proximity clusters [10]. Interestingly, recent studies reported rapid movements (snaps) of the chromosomal loci [19] and fast longitudinal density waves fluxing forth and back along the nucleoid that are independent of the ongoing replication [20]. An important future direction of studies is the elucidation of relationships between the chromosomal dynamics and different levels of structural organization, aiming at integration of these dynamical/organizational features with regulation of genetic function.

One promising approach is the determination of chromosomal domains as discrete structural-functional units distinctly responding to particular TFs (or combinations thereof). In this respect the best candidates are the global TFs, such as the highly abundant nucleoidassociated proteins (NAPs) in bacteria. The NAPs can regulate the activities of individual genes acting as bona fide TFs [21,22] but they can also bind at numerous genomic sites in a quasi-continuous manner with a wide range of affinities (spanning three orders of magnitude) and so modulate the chromosomal dynamics [23,24]. Such an integrative approach has been conducted recently in $E$. coli cultures during the growth cycle [25], and in the plant pathogen Dickeya dadantii, exposed to environmental stress [26]. The chromosomal domains have been identified on the basis of physical properties of the expressed sequences such as their dynamical behavior (preferred supercoiling regimen) thermodynamic stability (average negative melting energy), and spatial orientation in the genome (leading/lagging strand bias). It turned out that in $D$. dadantii the domains are formed transiently in response to the environmental stress, whereby it was possible to identify unique couplings between the dynamical and physicochemical properties of the expressed sequences, their functional content and the impacts of major NAPs, such as FIS (factor for inversion stimulation) and H-NS (histone-like nucleoid structuring protein). In particular, FIS activated the genes requiring high negative supercoiling of the DNA and mostly encoded on the leading strand, whereas H-NS repressed the genes requiring DNA relaxation that were preferentially encoded on the lagging strand [26]. Furthermore, since the domains were identified on the basis of expressed sequences, it was possible to link their physical characteristics to harbored genetic function. More specifically, chromosomal domains formed in response to particular stress were found to express different adaptation traits and virulence determinants, such that their transient activation mediated by global TFs conferred also the ability to cope with a specific challenge (Figure 1) [22,26-29].

Application of integrative approach enabled to reconstruct the pathogenicity process of $D$. dadantii in unprecedented detail. It turned out that this plant pathogenic bacterium uses transient organization of the chromosomal structural-functional domains under hostile conditions as a means to successfully invade and colonize its host. It thus appears that the rich information provided by such an integrative

*Corresponding author: Patrick Sobetzko, Philipps-Universität Marburg LOEWE-Center for Synthetic Microbiology, Marburg, Germany, Tel: +496421-28-22261; E-mail: Patrick.Sobetzko@synmikro.uni-marburg.de

Received: June 15, 2015; Accepted: June 24, 2015; Published June 26, 2015

Citation: Reverchon S, Sobetzko P, Nasser G, Muskhelishvili W (2015) Rethinking the Bacterial Genetic Regulation. Biochem Anal Biochem 4: 193. doi:10.4172/21611009.1000193

Copyright: ( 2015 Reverchon S, et al. This is an open-access article distributed under the terms of the Creative Commons Attribution License, which permits unrestricted use, distribution, and reproduction in any medium, provided the original author and source are credited. 


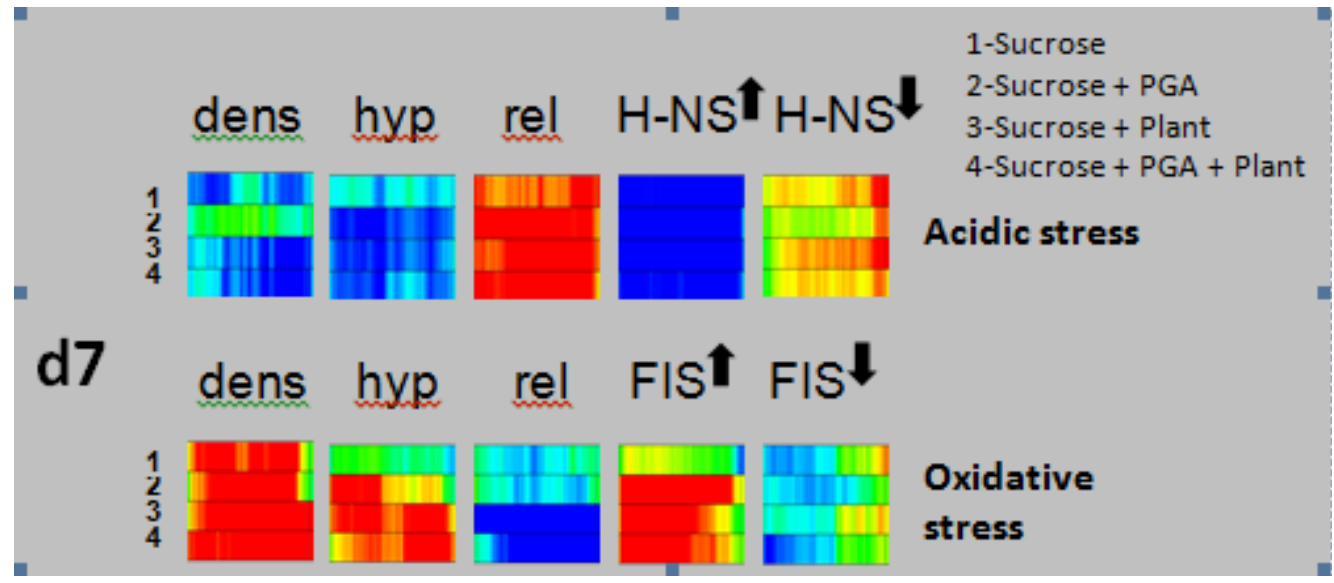

Figure 1: Response of the chromosomal domain d7 (indicated as a square composed of for horizontal rods corresponding to different growth media as numbered) spanning $560 \mathrm{~kb}$ of $D$. dadantii chromosome to oxidative and acidic stress involves sequences with different couplings of the parameters [26]. The colors indicate parameter values (red for high, and blue for low). During acidic stress encountered initially in the plant, the domain d7 demonstrates low gene expression density (dens) in combination with de-repression of the genes requiring DNA relaxation (rel) by H-NS (downward arrow). However, under these conditions the domain expresses the cfa gene involved in cell wall stabilisation in adaptation to acidic stress [22,27]. Oxidative stress following the acidic stress as a defense response of the plant increases the gene expression density of genes in d7. These genes require high levels of negative supercoiling (hyp) and are activated by FIS (upward arrow). Under these conditions the domain expresses the fliF- $R$ and cheRBYZ genes involved in motility and chemotaxis and supporting plant colonization, as well as pel virulence genes involved in plant cell wall degradation $[28,29]$. Thus the expression of these specific adaptation/virulence genes is dictated by peculiar coupling of DNA sequence parameters involved in formation of the entire "stress-response" domain.

approach is crucial for identification of new adaptation and virulence traits and designing tools for their targeted inactivation.

Whether the revealed mechanism of induction of transient chromosomal structural-functional domains harboring distinct adaptation/virulence functions is used as a means of adaptation by bacterial organisms in general remains to be elucidated, but since the changes of DNA supercoiling and modulatory effects of the NAPs are employed by most of the known bacterial pathogens [30,31], it is to be expected that the unveiled organizational principle will be widespread.

\section{References}

1. Roca I, Akova M, Baquero F, Carlet J, Cavaleri M, et al. (2015) The global threat of antimicrobial resistance: science for intervention. New Microbes New Infect 6: 22-29.

2. Czajkowski R, Pérombelon MCM, van Veen JA, van der Wolf JM (2011) Control of blackleg and tuber soft rot of potato caused by Pectobacterium and Dickeya species: a review. Plant Pathol 60: 999-1013.

3. Mansfield J, Genin S, Magori S, Citovsky V, Sriariyanum M, et al. (2012) Top 10 plant pathogenic bacteria in molecular plant pathology. Mol Plant Pathol 13: 614-629.

4. Conway T, Creecy JP, Maddox SM, Grissom JE, Conkle TL, et al. (2014) Unprecedented high-resolution view of bacterial operon architecture revealed by RNA sequencing. MBio 5: e01442-01414.

5. Jeong KS, Ahn J, Khodursky AB (2004) Spatial patterns of transcriptional activity in the chromosome of Escherichia coli. Genome Biol 5: R86.

6. Junier I and Rivoire O (2015) Conserved units of co-expression in bacterial genomes: an evolutionary insight into gene regulation.

7. Postow L, Hardy CD, Arsuaga J, Cozzarelli NR (2004) Topological domain structure of the Escherichia coli chromosome. Genes Dev 18: 1766-1779.

8. Meyer S, Beslon G (2014) Torsion-mediated interaction between adjacen genes. PLoS Comput Biol 10: e1003785

9. Sobetzko P, Muskhelishvili G (2015) Transcription-coupled DNA supercoiling dictates the chromosomal arrangement of bacterial genes. Nucleic Acids Res 20(3): 396-403.

10. Marr C, Geertz M, Hütt MT, Muskhelishvili G (2008) Dissecting the logical types of network control in gene expression profiles. BMC Syst Biol 2: 18.
11. Sonnenschein N, Geertz M, Muskhelishvili G, Hütt MT (2011) Analog regulation of metabolic demand. BMC Syst Biol 5: 40.

12. Niki H, Yamaichi $Y$, Hiraga S (2000) Dynamic organization of chromosomal DNA in Escherichia coli. Genes Dev 14: 212-223.

13. Berger M, Farcas A, Geertz M, Zhelyazkova P, Brix K, et al. (2010) Coordination of genomic structure and transcription by the main bacterial nucleoid-associated protein HU. EMBO Rep 11: 59-64.

14. Valens M, Penaud S, Rossignol M, Cornet F, Boccard F (2004) Macrodomain organization of the Escherichia coli chromosome. EMBO J 23: 4330-4341.

15. Képès $F(2004)$ Periodic transcriptional organization of the E.coli genome. J Mol Biol 340: 957-964.

16. Scolari VF, Bassetti B, Sclavi B, Lagomarsino MC (2011) Gene clusters reflecting macrodomain structure respond to nucleoid perturbations. Mol Biosyst 7: 878-888.

17. Mathelier A, Carbone A (2010) Chromosomal periodicity and positional networks of genes in Escherichia coli. Mol Syst Biol 6: 366.

18. Ma Q, Yin Y, Schell MA, Zhang H, Li G, et al. (2013) Computational analyses of transcriptomic data reveal the dynamic organization of the Escherichia coli chromosome under different conditions. Nucleic Acids Res 41: 5594-5603.

19. Javer A, Kuwada NJ, Long Z, Benza VG, Dorfman KD, et al. (2014) Persistent super-diffusive motion of Escherichia coli chromosomal loci. Nat Commun 5: 3854

20. Fisher JK, Bourniquel A, Witz G, Weiner B, Prentiss M, et al. (2013) Fourdimensional imaging of $\mathrm{E}$. coli nucleoid organization and dynamics in living cells. Cell 153: 882-895.

21. Lautier T, Blot N, Muskhelishvili G, Nasser W (2007) Integration of two essentia virulence modulating signals at the Erwinia chrysanthemi pel gene promoters: a role for Fis in the growth-phase regulation. Mol Microbiol 66: 1491-1505.

22. Reverchon S, Nasser W (2013) Dickeya ecology, environment sensing and regulation of virulence programme. Environ Microbiol Rep 5: 622-636.

23. Travers A, Muskhelishvili G (2005) Bacterial chromatin. Curr Opin Genet Dev 15: $507-514$

24. Dorman CJ (2013) Genome architecture and global gene regulation in bacteria: making progress towards a unified model? Nat Rev Microbiol 11: 349-355.

25. Sobetzko P, Glinkowska M, Travers A, Muskhelishvili G (2013) DNA thermodynamic stability and supercoil dynamics determine the gene expression program during the bacterial growth cycle. Mol Biosyst 9: 1643-1651.

26. Jiang X, Sobetzko P, Nasser W, Reverchon S, Muskhelishvili G (2015) 
Citation: Reverchon S, Sobetzko P, Nasser G, Muskhelishvili W (2015) Rethinking the Bacterial Genetic Regulation. Biochem Anal Biochem 4: 193. doi:10.4172/2161-1009.1000193

Page 3 of 3

Chromosomal "stress-response" domains govern the spatiotemporal expression of the bacterial virulence program. MBio 6: e00353-00315.

27. Nasser W, Reverchon S (2002) H-NS-dependent activation of pectate lyases synthesis in the phytopathogenic bacterium Erwinia chrysanthemi is mediated by the PecT repressor. Mol Microbiol 43: 733-748.

28. Ouafa ZA, Reverchon S, Lautier T, Muskhelishvili G, Nasser W (2012) The nucleoid-associated proteins $\mathrm{H}-\mathrm{NS}$ and FIS modulate the DNA supercoiling response of the pel genes, the major virulence factors in the plant pathogen bacterium Dickeya dadantii. Nucleic Acids Res 40: 4306-4319.
29. Duprey A, Reverchon S, Nasser W (2014) Bacterial virulence and Fis: adapting regulatory networks to the host environment. Trends Microbiol 22: 92-99.

30. Dorman CJ (2011) Regulation of transcription by DNA supercoiling in Mycoplasma genitalium: global control in the smallest known self-replicating genome. Mol Microbiol 81: 302-304.

31. Dorman CJ (2014) Function of nucleoid-associated proteins in chromosome structuring and transcriptional regulation. J Mol Microbiol Biotechnol 24: 316-331. 